\title{
ADAPTACIÓN DE INDICADORES DE SUSTENTABILIDAD EN ÁREAS DE CRECIMIENTO URBANO Análisis de caso en el municipio del Pilar, Argentina
}

\author{
ADAPTATION OF SUSTAINABILITY INDICATORS IN \\ URBAN GROWTH AREAS \\ Case analysis in Pilar county, Argentina
}

\author{
A. Gabriel Mancuello \\ Universidad de Buenos Aires. Argentina \\ gabriel.mancuello@fadu.uba.ar
}

\section{RESUMEN}

El crecimiento de las ciudades, con una tendencia exponencial de expansión territorial y en un contexto actual de creciente urbanización de la población a nivel global, pone en relevancia la necesidad de un análisis de la sustentabilidad con la que se está dando este proceso. Mientras que existen diversas metodologías de análisis mediante indicadores de sustentabilidad en casos europeos, las mismas no se presentarían pertinentes para un análisis en un contexto latinoamericano. El presente trabajo indaga en el análisis de pertinencia y posible adaptación conceptual de indicadores elaborados por la Agencia de Ecología Urbana de Barcelona, tanto en los estándares propuestos, como en su relación con la situación actual y la situación postulada por la normativa urbana, proponiendo finalmente lineamientos para el análisis de la sustentabilidad de estas áreas de crecimiento.

Palabras clave: Crecimiento urbano, indicadores, sustentabilidad, urbanización.

Líneas de investigación: 1: Ciudad y proyecto.

Tema: Estudios metropolitanos y territoriales.

\section{ABSTRACT}

\begin{abstract}
The growth of cities, with an exponential trend of territorial expansion and a current context of increasing urbanization of the population at a global level, highlights the need for an analysis of the sustainability which this process is taking place. While there are several analysis methodologies through sustainability indicators in European cases, they would not be presented relevant for an analysis in a Latin American context. The present work investigates the analysis of relevance and possible conceptual adaptation of urban morphology indicators prepared by the Urban Ecology Agency of Barcelona, both in the proposed standards as its relation to the current situation and the situation postulated by urban regulations, finally proposing guidelines for the analysis of the sustainability of these growth areas.
\end{abstract}

Keywords: Urban growth, indicators, sustainability, urbanization.

Research line: 1: City and project.

Topic: Metropolitan and territorial studies. 


\section{SÃO PAULO15 17 LISBOA $25 \sim 26$ JUN 2020}

\section{Introducción y estado actual del conocimiento del tema}

\subsection{Crecimiento urbano}

El creciente proceso de urbanización de la población a nivel global que muestran las estadísticas demográficas en los últimos años (Demographia, 2019), ha impulsado el crecimiento de las ciudades con una tendencia creciente de desarrollo de urbanizaciones con gran consumo de suelo y baja densidad poblacional. Estas tendencias ponen en crisis la sostenibilidad de este proceso. Diversos estudios dan cuenta de esta tendencia en las ciudades, como el análisis de expansión urbana realizado por el Lincoln Institute of Land Policy en áreas metropolitanas a nivel mundial (Angel et al., 2016).

En Latinoamérica, que presenta uno de los mayores índices de urbanización de la población a nivel global, esta problemática adquiere mayor relevancia. En la Argentina en particular, un estudio en los principales aglomerados urbanos país (Lanfranchi et al., 2018) comprueba un crecimiento territorial en aumento en los últimos años, con una tendencia decreciente en la densidad poblacional de estas ciudades. Según este estudio, en la Región Metropolitana de Buenos Aires (RMBA), el crecimiento urbano registrado en los períodos 2006-2016 se debió en un 46\% a urbanizaciones cerradas, una tipología correlacionada directamente con el crecimiento difuso y la segregación urbana.

Aunque existe cierto consenso respecto a los beneficios de la ciudad densa, se presentan también algunos aspectos negativos que conlleva la densidad (García, 1999), por los que algunos autores sostienen que no debería plantearse una dicotomía entre ciudad densa y ciudad dispersa, sino un análisis en otros términos (Farguell, 2007). Los patrones de crecimiento urbano demostrados en los últimos años requieren de una revisión conceptual para establecer cuáles son los estándares de densidad o dispersión a los que se aspira, para poder establecer finalmente su sustentabilidad.

El análisis del crecimiento urbano, puede ser clasificado por su extensión territorial densa o difusa, pero también por las formas que lo materializan. En este sentido, es de interés la metodología propuesta Sola Morales (1997) para esta clasificación. El autor define distintas formas de crecimiento urbano mediante el análisis de tres variables básicas de este proceso: urbanización, parcelación y edificación. Esta clasificación aplicada en Argentina se propone en esta investigación como base para la delimitación territorial que permite la evaluación del crecimiento urbano.

En cuanto al crecimiento por densificación el trabajo realizado por Diez (1996) resulta pertinente para poder establecer relaciones entre el estado actual de la edificación, la normativa potencial permitida y el tejido resultante en el proceso.

\subsection{Indicadores de sustentabilidad}

Existen diversos estudios sobre el crecimiento urbano realizados mediante indicadores cuantitativos de expansión territorial, densidad y población, y otros que abordan la escala metropolitana con indicadores de sustentabilidad aplicados desde la interpretación de imágenes satelitales, como el reciente estudio del BID a nivel latinoamericano de la calidad urbana (Poskus et al., 2018). De esta forma, mientras que el análisis metropolitano ha sido ampliamente abordado, no lo ha sido así la escala de sector. Por lo tanto, esta investigación plantea indagar en una metodología que permita caracterizar la heterogeneidad y medir la 


\section{SÃO PAULO15 17 LISBOA $25 \sim 26$ JUN 2020}

sustentabilidad de las distintas tipologías urbanas que materializan el crecimiento territorial. Esta exploración permitiría una posible evaluación de cada una de estas tipologías, mediante otras variables urbanísticas, con mayor grado de especificidad.

El análisis de tipologías urbanas en sectores acotados y delimitados permitiría realizar una evaluación mediante indicadores de sustentabilidad para la caracterización de cada sector. Para su aplicación, de destaca el marco teórico propuesto por el urbanismo ecológico (Rueda, 2010) desarrollado en la Agencia de Ecología Urbana de Barcelona (AEUB) y los distintos indicadores propuestos para mesurar el alcance de las metas (Rueda, 2018). Aunque el autor ha hecho aportes a la esfera gubernamental para una adaptación al contexto particular de la ciudad de Buenos Aires (Rueda, Enero de 2018), este incipiente trabajo no comprende aún el análisis de entornos suburbanos propios del área metropolitana.

\section{Objetivos e hipótesis de investigación}

El presente trabajo se propone indagar en la pertinencia y adaptación de indicadores elaborados por la AEUB, tanto en su metodología como en los estándares utilizados, para la evaluación de distintas tipologías de crecimiento urbano en los casos de estudio seleccionados. El mismo se encuadra en un proyecto de investigación que se viene desarrollando en diferentes etapas desde el año 2014 y que se propone identificar, observar y comparar distintas formas de crecimiento urbano, con la finalidad de evaluar la sostenibilidad del crecimiento y proponer lineamientos para optimizar su control.

La clasificación tipológica del crecimiento urbano plantea como hipótesis principal, que las formas identificadas en Barcelona por Solá Morales (1997) pueden aplicarse en la RMBA para casos de crecimiento por extensión mediante una interpretación conceptual. Se plantea además, que cada una de estas formas por sus características urbanísticas específicas de conformación temporal, presentaría evaluaciones distintas respecto a su sustentabilidad.

El trabajo analítico y de evaluación de la sustentabilidad de cada forma de crecimiento plantea realizar una evaluación aplicando indicadores de sustentabilidad de la AEUB ya validados en otros contextos. La hipótesis de trabajo es que, para realizar esta evaluación de sustentabilidad en áreas suburbanas y periurbanas en un contexto latinoamericano, se requiere ineludiblemente de una adaptación metodológica y conceptual, no sólo de la forma de medición del indicador sino también del estándar que se toma como ideal, para permitir así una mejor aproximación a la realidad propia local. Se parte de la hipótesis de que los mismos son adaptables para ser aplicados a entornos locales acotados, temporal y espacialmente, siendo necesario reconsiderar los estándares aplicables a la realidad latinoamericana. En función de los resultados obtenidos con los indicadores se plantearán reflexiones para su adaptación al contexto local.

Las preguntas de investigación a responder en función de los indicadores aplicados son: ¿Qué estándares son los apropiados en función de un modelo de ciudad en los casos de estudio seleccionados?; ¿Qué relación existe entre las formas de crecimiento extensivo y el crecimiento por densificación?; ¿Qué características condicionan la posible modificación de estos indicadores hacia el modelo de ciudad deseado? 


\section{SÃO PAULO $15 \sim 17 \cdot$ LISBOA $25 \sim 26$ JUN 2020 \\ Seminário Internacional de Investigação em Urbanismo \\ Seminario Internacional de Investigación en Urbanismo}

\section{Desarrollo analítico}

\subsection{Recorte territorial de la investigación}

Como primera aproximación al a la problemática del crecimiento urbano por extensión se tomó como recorte de análisis el Municipio del Pilar, que se encuentra en el tercer cordón de la RMBA. Este municipio ha registrado en las últimas tres décadas un crecimiento exponencial en cuanto a consumo de suelo producto de un proceso de dinámicas de crecimiento metropolitano que lo fueron incorporando a la conurbación de la ciudad de Buenos Aires. Por otro lado, el crecimiento ha sido paradigmático en cuanto a las tipologías que lo materializaron, principalmente con la proliferación de urbanizaciones cerradas, en un contexto de segregación social mediante enclaves urbanos.

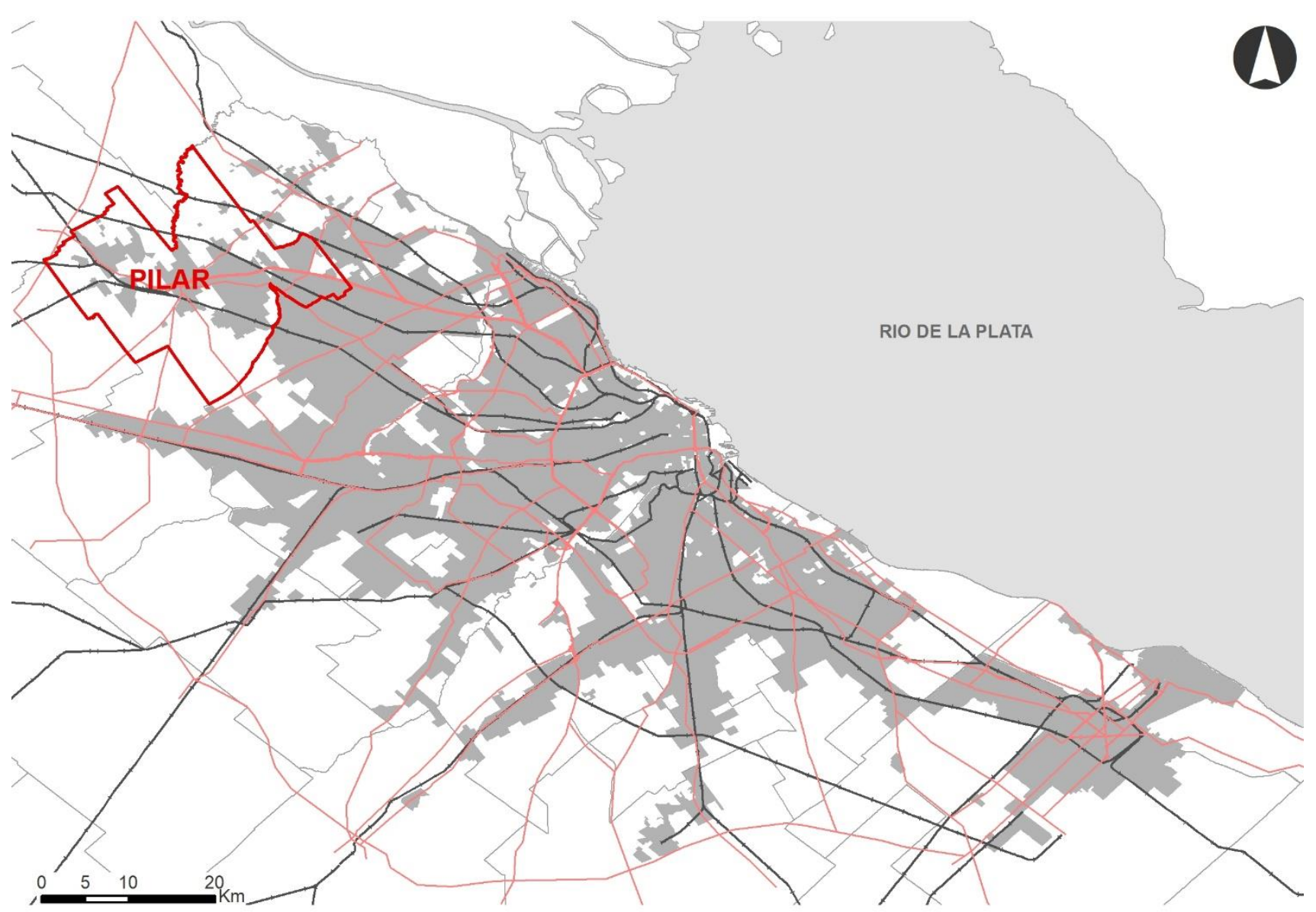

Figura 1. Municipio del Pilar en la región metropolitana de Buenos Aires. Fuente: Elaboración propia. 


\section{SÃOPAULO15 $17 \cdot$ LISBOA $25 \sim 26$ JUN 2020}

Seminário Internacional de Investigação em Urbanismo

\subsection{Clasificación de formas del crecimiento urbano extensivo}

\subsubsection{Metodología}

Mientras que en la actualidad el análisis predominante del crecimiento urbano toma la extensión territorial o el crecimiento de la mancha urbana, el enfoque de este proyecto propone el análisis e identificación de las tipologías urbanísticas con que se materializa este proceso de crecimiento mediante la metodología propuesta por Solá Morales (1997).

Esta metodología tipifica los procesos de crecimiento urbano como conjunto de operaciones materiales de construcción de la ciudad, según el orden y la importancia de cada una de estas operaciones: parcelación, urbanización y edificación. La parcelación $(P)$, entendida como transformación de suelo rústico en urbano y como atribución de uso a un suelo; la urbanización (U) como operación de construcción de elementos físicos colectivos de la ciudad y la edificación (E), como construcción del tejido urbano.

Las distintas combinaciones de operaciones materiales definen las distintas formas de crecimiento posibles. Como recorte teórico e instrumental en esta línea de investigación se tomaron únicamente los procesos que el autor agrupa como reconocidos por el planeamiento (esto es los procesos de la ciudad formal):

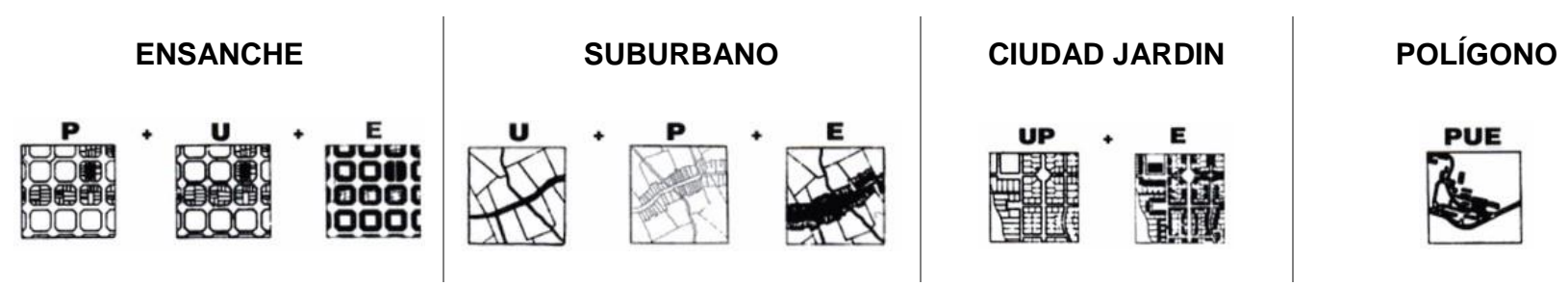

Figura 2. Procesos de crecimiento urbano reconocidos por el planeamiento. Fuente: (Solá Morales, 1997).

\subsubsection{Adaptación de marco teórico e identificación de formas de crecimiento en el municipio del Pilar}

El reconocimiento de las distintas formas de crecimiento urbano en el municipio del Pilar, requiere, con excepción del caso del polígono, de ciertas adaptaciones conceptuales previas respecto a tipificación realizada por Solá Morales en Barcelona

El Ensanche caracterizado por un tipo edificatorio complejo, con usos diversos de suelo, capacidad de transformación permanente y ordenación en malla o cuadrícula que permitiría su expansión, se encuentra en el caso del municipio del Pilar asociado al casco de fundación de la ciudad. El mismo, con una cuadrícula definida, carácter de centralidad urbana, diversidad de usos y una normativa que permite su densificación, se encuadra dentro de esta tipificación.

El caso de la Ciudad Jardín, se encuentra en el municipio del Pilar en la forma de enclave urbano como una urbanización cerrada. En ambos casos los mismos responden a un proceso de constitución en donde la edificación (E) se materializa una vez que se encuentra presente el parcelamiento $(P)$ y la red de servicios de infraestructura $(U)$. 


\section{SÃOPAULO15 $17 \cdot$ LISBOA $25 \sim 26$ JUN 2020 \\ Seminário Internacional de Investigação em Urbanismo \\ Seminario Internacional de Investigación en Urbanismo}

En el suburbano el proceso de urbanización arranca con una infraestructura (U) existente (en el caso de estudio seleccionado la ruta nacional 8), pero a diferencia de Barcelona el completamiento de la urbanización $(U)$, se prolonga en el tiempo (redes de servicios de infraestructura), superponiéndose con la parcelación y edificación, pero manteniendo la secuencia U-P-E.

\subsection{Selección y delimitación de los casos de estudio}

Las distintas formas de crecimiento urbano, clasificadas en el municipio del Pilar en función del marco teórico, fueron definidas en el proyecto de investigación como unidades territoriales de crecimiento (UTC). Para el análisis de las características urbanas de las UTC se seleccionaron cuatro casos de estudio representativos de cada una de las formas de crecimiento urbano, en un área relativamente próxima para descartar la variable de su ubicación relativa, y operativamente facilitar el relevamiento posterior de datos.

La delimitación específica de los casos de estudios seleccionados representativos de cada forma de crecimiento se realizó en el caso del Ensanche y Suburbano mediante planos históricos (Figura 3) que permitieron reconstruir el trazado inicial del proceso de parcelación $(P)$.

Plano de mensura de Pilar de 1884

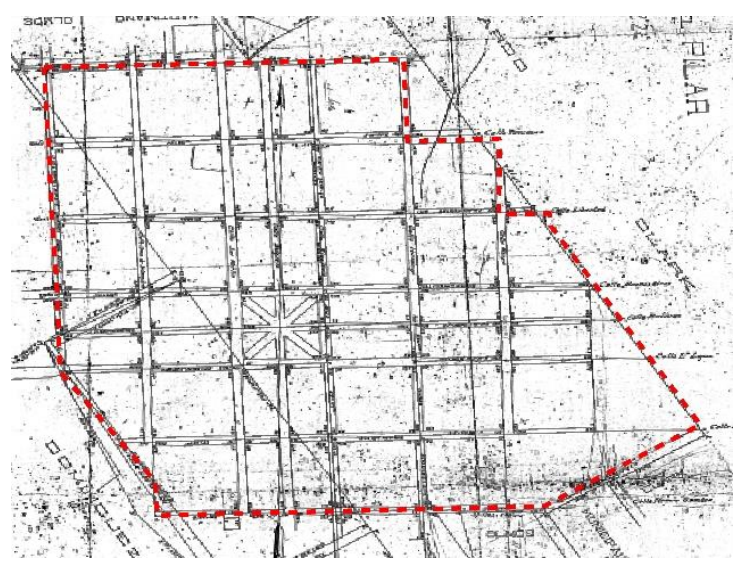

Pilar en la actualidad

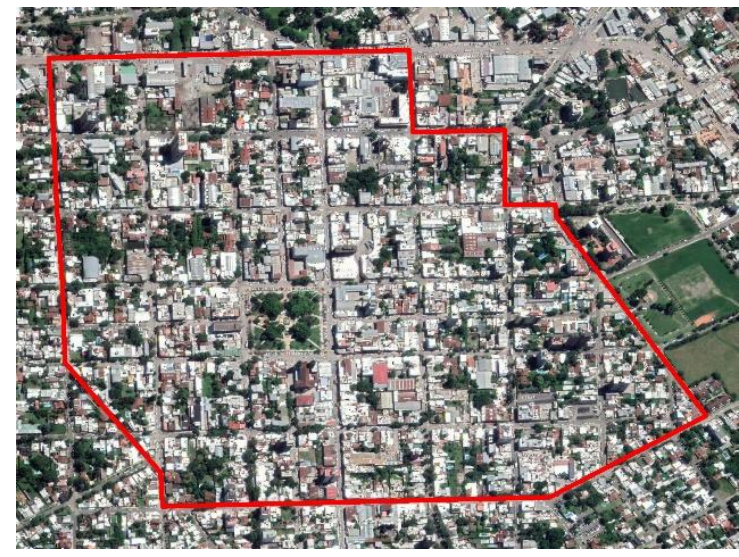

Figura 3. Delimitación de forma de crecimiento de Ensanche en el casco histórico de Pilar. Fuente: Elaboración propia.

En el caso del Polígono y la Ciudad Jardín, para la delimitación no fue necesaria la consulta a planos históricos ya que, por sus características propias, estas se encuentran delimitadas desde el mismo proyecto en el caso del Polígono y como urbanización cerrada en el caso de la Ciudad Jardín.

UTC seleccionadas como caso de estudio:

- Ensanche: Casco histórico de Pilar

- Suburbano: Parcelamiento del barrio Pilarica

- Ciudad Jardín: Barrio Cerrado "El Portal"

- Polígono: Conjunto habitacional (Barrio Vicente Lopez) 


\section{SÃO PAULO15 17 LISBOA $25 \sim 26$ JUN 2020}

Seminário Internacional de Investigação em Urbanismo

Seminario Internacional de Investigación en Urbanismo

Clasificación de los casos de estudio seleccionados según su estado:

\begin{tabular}{l|l}
\hline \multicolumn{1}{c|}{ UTC } & \multicolumn{1}{|c}{ ESTADO (según normativa) } \\
\hline ENSANCHE & EN DENSIFICACIÓN \\
\hline SUBURBANO & INCOMPLETO \\
\hline CIUDAD JARDÍN & COMPLETO \\
\hline POLÍGONO & COMPLETO \\
\hline
\end{tabular}

Tabla 3. Clasificación de UTC según el estado de consolidación. Fuente: Elaboración propia.

\subsection{Aplicación de indicadores de sustentabilidad (AEUB)}

La metodología implementada desde la AEUB para la aplicación de indicadores se realiza en recortes territoriales más amplios a los abordados en esta investigación, por lo que utilizan de base una grilla modular de $200 \times 200$ metros. En los sectores de menor escala, como se pudo observar en etapas anteriores de la presente investigación, la aplicación de indicadores precisa de otras formas de medición del desempeño de cada unidad territorial que se ajusten a su tipología (Compagnoni et al., 2018), ya que la grilla desfasada de las unidades de análisis no permite una correcta lectura del desempeño del indicador. En este sentido, la aplicación de indicadores se realiza en este trabajo de manera global según el límite propio de cada unidad territorial de crecimiento y no desde una grilla homogénea.

Para el cálculo de los indicadores incluidos en esta presentación se utilizaron los datos presentados (tabla 4), obtenidos tanto por relevamiento como por interpretación de imágenes satelitales.

\begin{tabular}{lcrrrr}
\hline Datos de caracterización & UNIDAD & POLIGONO & ENSANCHE & SUBURBANO & C. JARDIN \\
\hline Superficie UTC & $\mathrm{m} 2$ & 7.937 & 564.987 & 364.573 & 62.832 \\
Viviendas & $\mathrm{un}$ & 84 & 2.157 & 336 & 54 \\
Volumen edificado & $\mathrm{m} 3$ & 17.964 & 1.307 .799 & 213.678 & 44.508 \\
Espacio público total & $\mathrm{m} 2$ & 2.604 & 142.612 & 102.574 & 21.520 \\
Superficie permeable & $\mathrm{m} 2$ & 1.459 & 89.604 & 273.303 & 41.611 \\
\hline
\end{tabular}

Tabla 4. Datos relevados para el cálculo de indicadores. Fuente: Elaboración propia. 


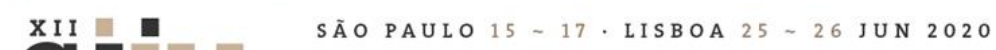

Seminário Internacional de

Seminario Internacional de Investigação em Urbanismo

\subsubsection{Densidad de viviendas}

El indicador de densidad de viviendas se propone cuantificar la cantidad de personas en un determinado recorte territorial que permita alcanzar una mínima masa crítica que incite intercambios y nuevas relaciones comunicativas entre personas, entes y actividades. De esta manera se permitiría desarrollar con eficiencia aquellas funciones urbanas ligadas a la movilidad sostenible y a la dotación de servicios tanto en el ámbito del transporte público, de las infraestructuras ligadas a los flujos metabólicos o de los equipamientos y servicios básicos.

Densidad de viviendas $=\frac{\text { Viviendas } * 10000}{\text { Superficie total UTC }\left(\mathrm{m}^{2}\right)}$
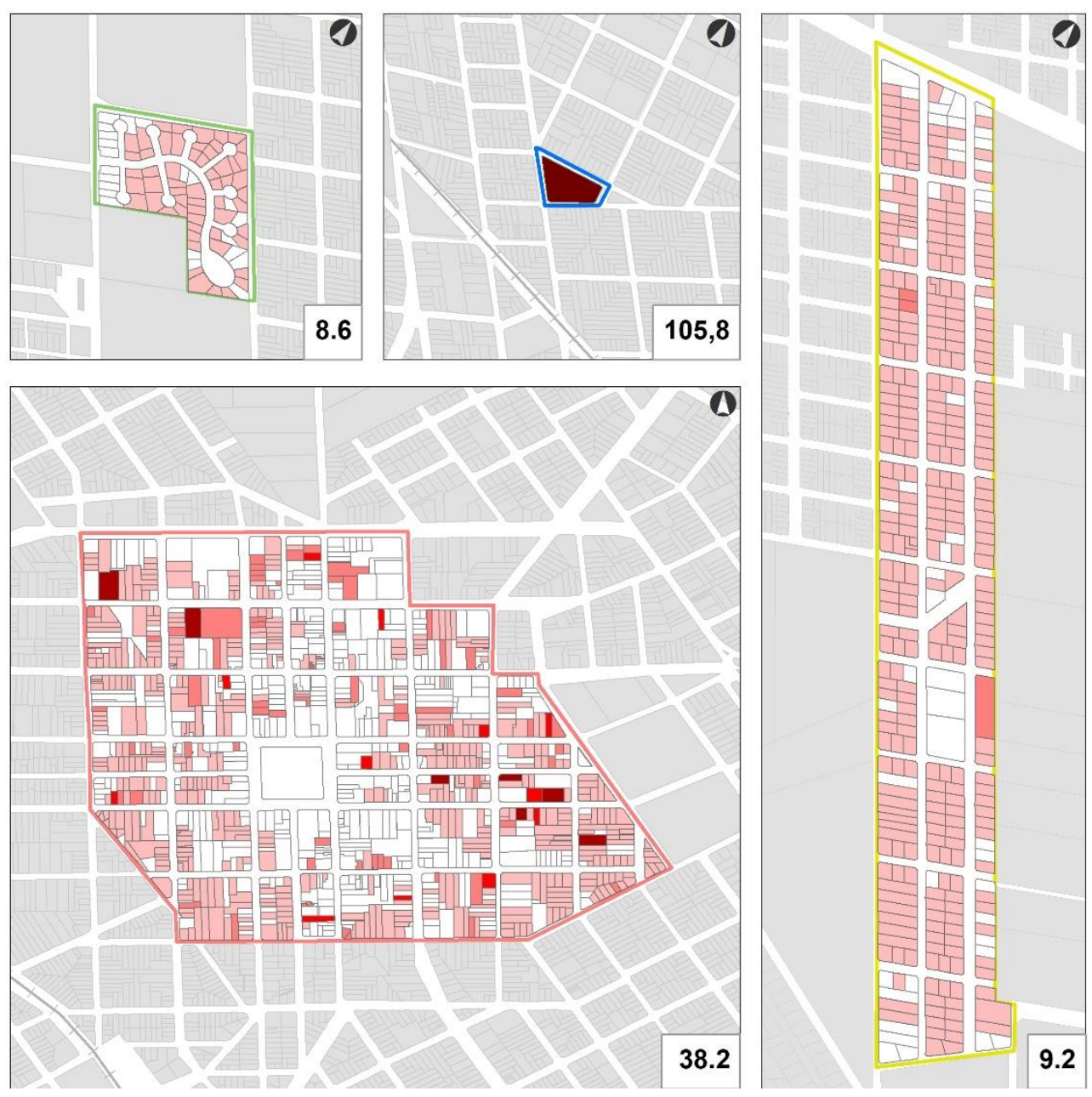
INDICADOR DENSIDAD DE VIVIENDAS Estándar $60 \mathrm{Viv} / \mathrm{Ha}$
CIUDAD JARDIN
POLÍGONO
105,8
ENSANCHE $\quad 38,2$
SUBURBANO
9,2

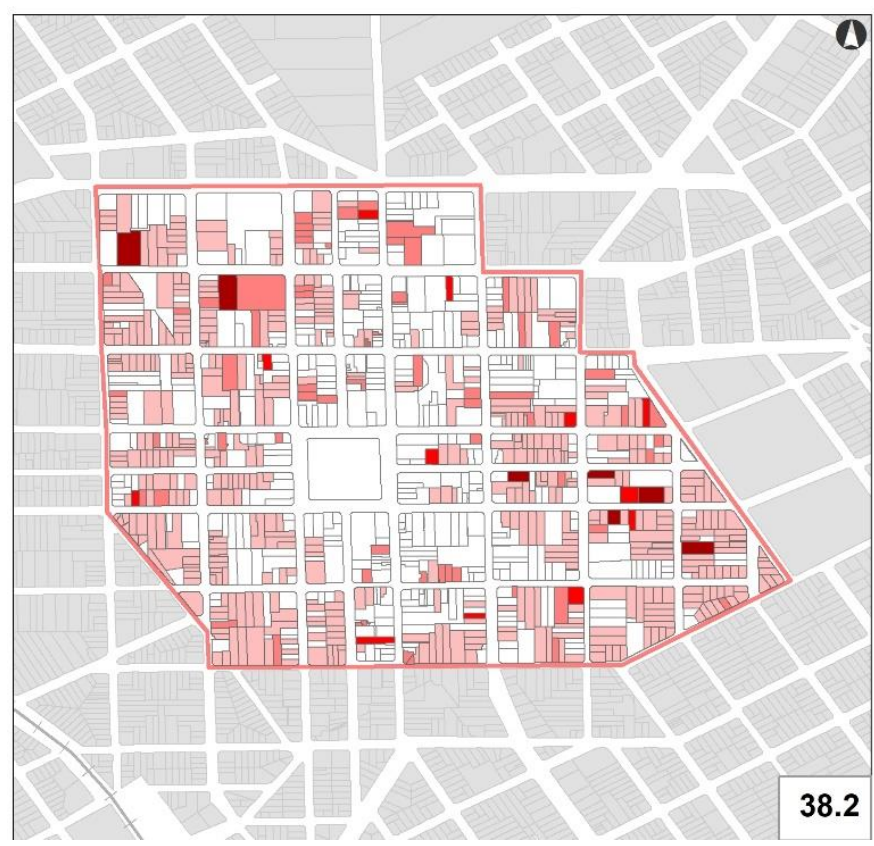

\section{(}

9.2

\section{REFERENCIAS}

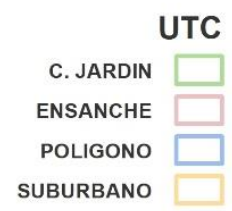

.Cant. de viv

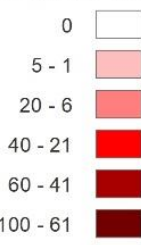

Figura 6. Indicador de densidad de viviendas. Fuente: Elaboración propia. 


\section{SÃO PAULO15 $\sim 17 \cdot$ LISBOA $25 \sim 26$ JUN 2020}

Seminário Internacional de

Seminario Internacional de Investigação em Urbanismo

\subsubsection{Compacidad absoluta}

El indicador de Compacidad absoluta cuantifica el grado de ocupación del territorio y su densidad edilicia tomando como estándar un modelo de ocupación compacto del territorio para buscar la eficiencia en el uso de los recursos naturales y disminuir la presión de los sistemas urbanos sobre los sistemas de apoyo.

Compacidad absoluta $=\frac{\text { Volumen edificado }\left(\mathrm{m}^{3}\right)}{\text { Superficie total UTC }\left(\mathrm{m}^{2}\right)}$
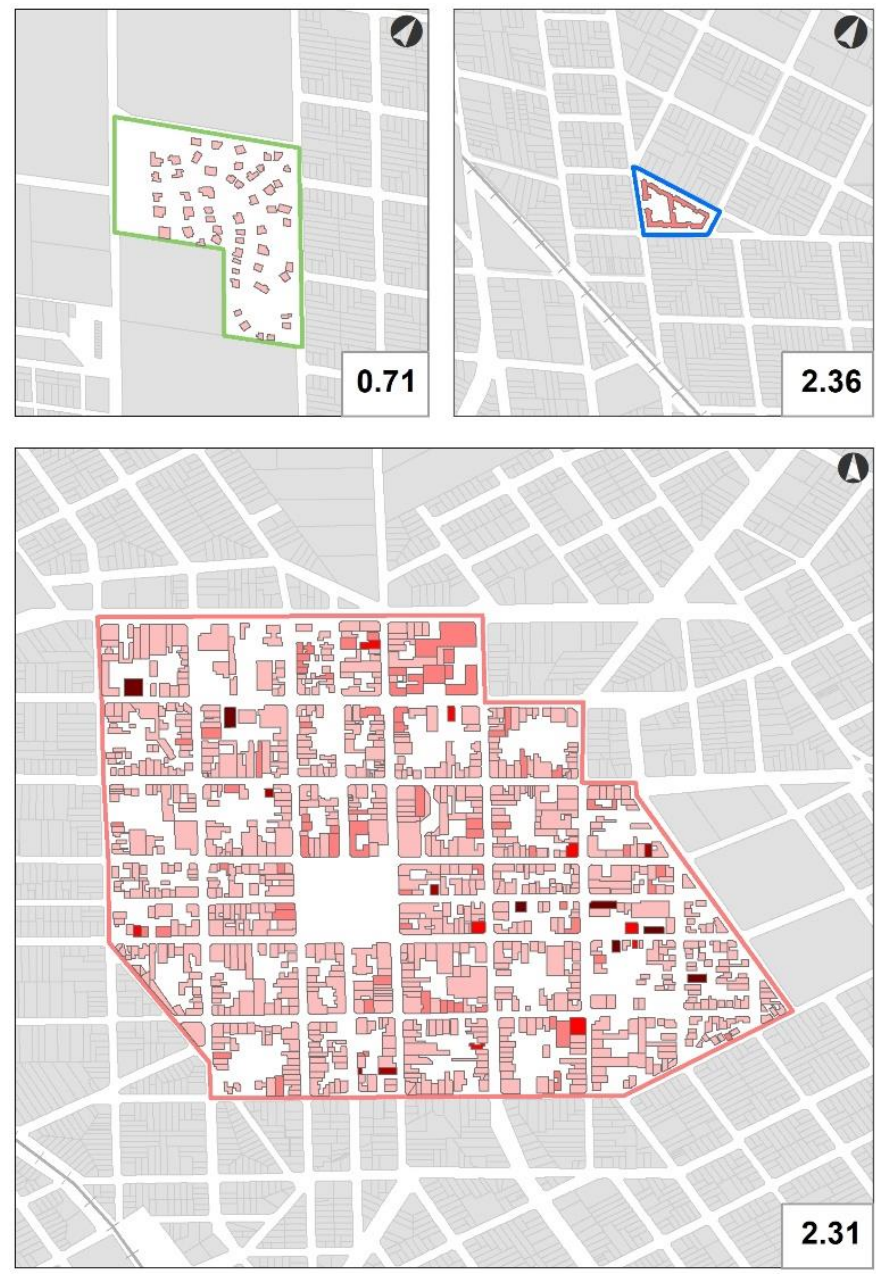

INDICADOR COMPACIDAD ABSOLUTA

Estándar $5 \mathrm{~m}$

CIUDAD JARDIN $\quad 0.71$

POLÍGONO 2.26

ENSANCHE $\quad 2.31$

SUBURBANO 0.59

\section{REFERENCIAS}

Altura en pisos

$0-1$

$2-1$

$5-3$

$8-6$

$10-9$

$14-11$

\section{UTC}

C. JARDIN

ENSANCHE

POLIGONO

SUBURBANO

0.59

Figura 7. Indicador de compacidad absoluta. Fuente: Elaboración propia. 


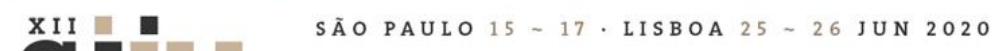

Seminário Internacional de Investigação em Urbanismo

\subsubsection{Compacidad corregida (adaptado)}

El indicador de compacidad corregida da una idea de la esponjosidad del tejido urbano para la consecución de actividades ligadas al espacio público. A diferencia de la compacidad absoluta que relaciona el volumen edificado y su relación con el espacio urbano (público o privado), la compacidad corregida relaciona el volumen edificado con el espacio público. Originalmente Rueda (2018) plantea esta relación con lo que el autor denomina espacio público de estancia, es decir, espacio público peatonal que posea un ancho específico de $5 \mathrm{~m}$, para permitir actividades de recreo y esparcimiento. Por una simplificación metodológica y posibilidades de obtención de información, en esta propuesta se va a tomar el espacio público total sin estas distinciones. De todas formas, a los fines de análisis del indicador los resultados mantienen su pertinencia.

Compacidad corregida $=\frac{\text { Volumen edificado }\left(\mathrm{m}^{3}\right)}{\text { Espacio público total }\left(\mathrm{m}^{2}\right)}$
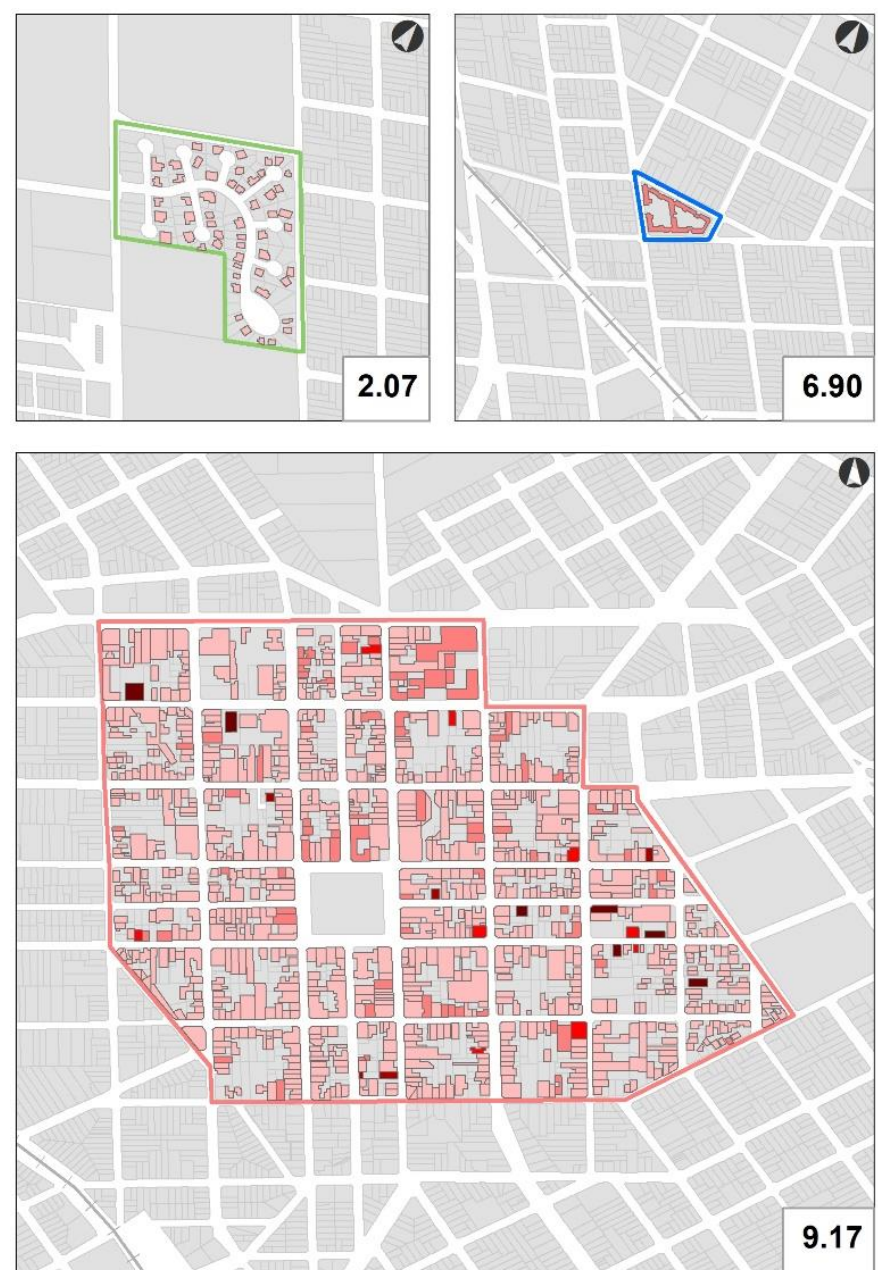

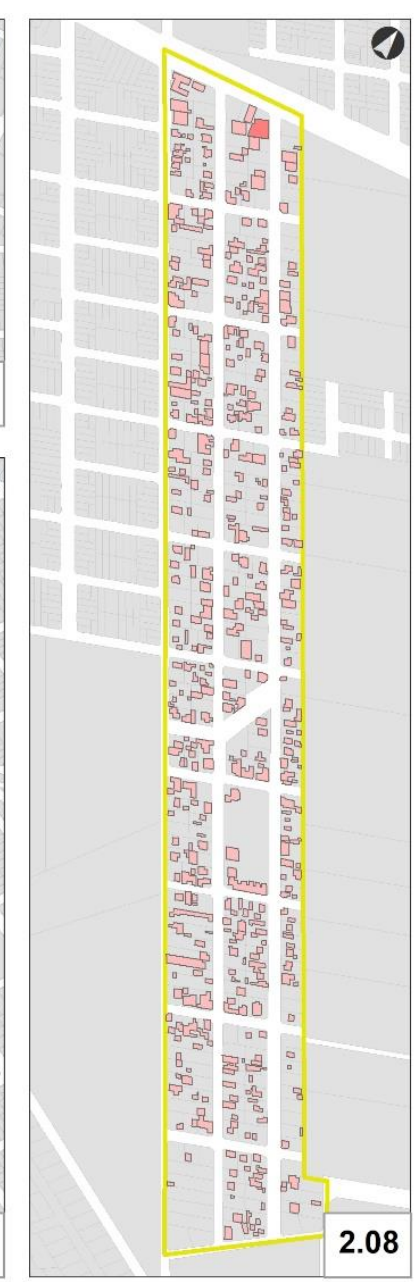

INDICADOR COMPACIDAD CORREGIDA Estándar 10 a $60 \mathrm{~m}$

CIUDAD JARDIN

2.07

POLIGONO $\quad 6.90$

ENSANCHE 9.17

SUBURBANO

2.08

REFERENCIAS

Altura en pisos

$0-1$

$2-1$

$5-3$

$8-6$

$10-9$

$14-11$

UTC

C. JARDIN

ENSANCHE

POLIGONO

SUBURBANO

Figura 8. Indicador de compacidad corregida. Fuente: Elaboración propia. 


\section{SÃO PAULO15 $\sim 17 \cdot$ LISBOA $25 \sim 26$ JUN 2020}

Seminário Internacional de

Seminario Internacional de Investigação em Urbanismo

\subsubsection{Permeabilidad urbana}

La proporción de suelo permeable en los tejidos urbanos debe garantizar la continuidad de las superficies verdes y el buen funcionamiento del sistema hídrico. El sellado y la impermeabilización del suelo de forma masiva frenan la posibilidad de vida vegetal y la aparición de multitud de organismos dependientes.

Permeabilidad urbana $=\frac{\text { Superficie total UTC }\left(\mathrm{m}^{2}\right)}{\text { Superficie permeable }\left(\mathrm{m}^{2}\right)}$
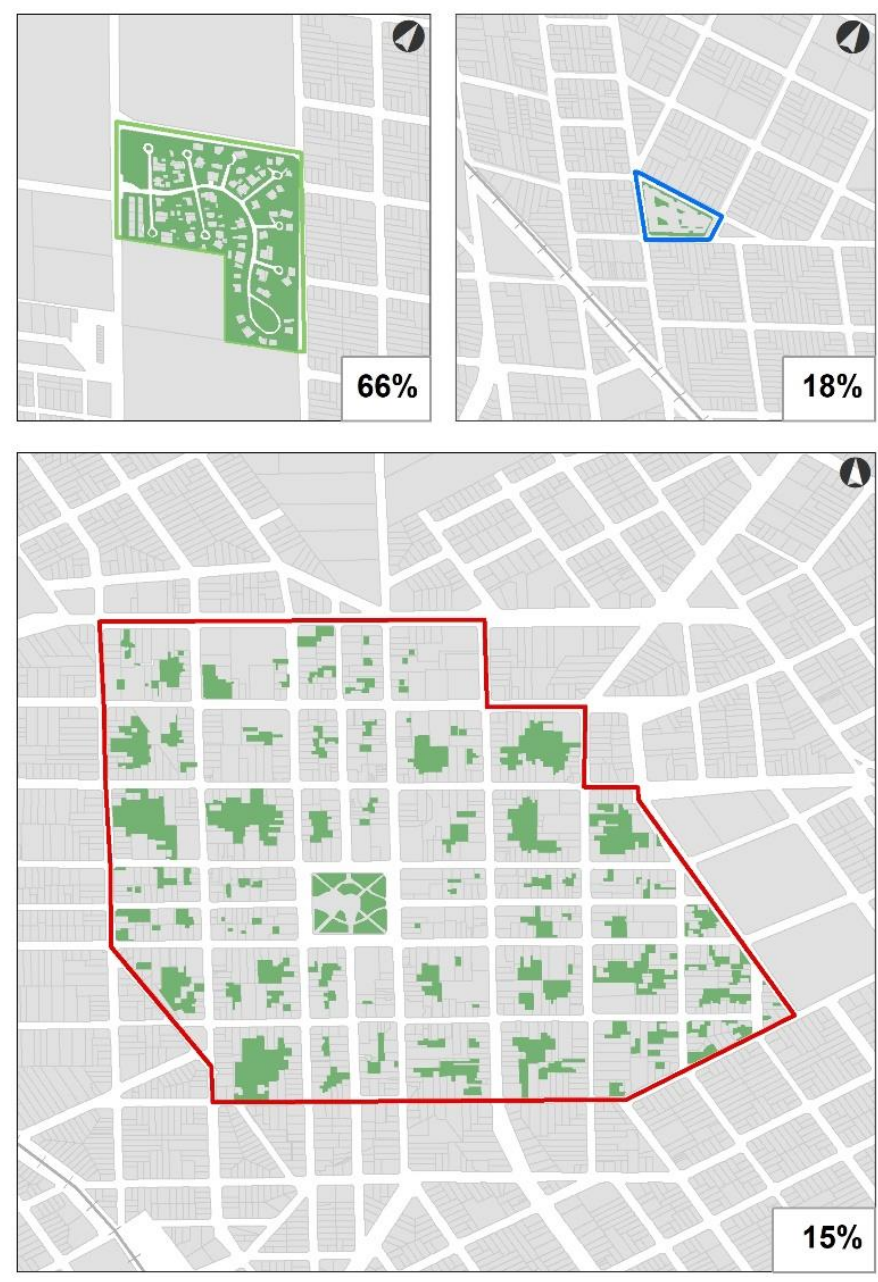

INDICADOR PERMEABILIDAD URBANA

Est. $>30 \%$ Sup. permeable

CIUDAD JARDIN $\quad 66,2 \%$

POLÍGONO $\quad 18,4 \%$

ENSANCHE $\quad 15,9 \%$

SUBURBANO $\quad 74,9 \%$

REFERENCIAS

Superficie permeable

UTC

C. JARDIN

ENSANCHE

POLIGONO

SUBURBANO

$74 \%$

Figura 9. Indicador de permeabilidad urbana. Fuente: Elaboración propia. 


\section{SÃO PAULO15 17 LISBOA $25 \sim 26$ JUN 2020}

\section{Reflexiones metodológicas y conceptuales}

\subsection{Estándares}

Las mediciones de los indicadores nos permiten tener un valor cuantitativo de una determinada variable para cada UTC, pero es su relación con el estándar ideal lo que permite realmente su caracterización. La AEUB desarrolló el sistema de indicadores para casos de sector en un contexto europeo principalmente para grandes ciudades, pero como cada contexto urbano requiere establecer sus propios estándares, se realizó una indagación de estándares establecidos por normativa y estándares teóricos de tipologías paradigmáticas.

Si tomamos los estándares establecidos por la normativa territorial, podemos establecer una comparación entre los valores actuales de cada UTC para el indicador, el estándar de sostenibilidad establecidos por la AEUB y los estándares establecidos como potenciales por normativa por el gobierno local del municipio. Particularmente en el Municipio del Pilar recientemente se ha aprobado el Código de Ordenamiento Territorial (COT) (2019) por lo que estos valores se encuentran actualizados al modelo de ciudad definido recientemente. Para el caso del indicador de densidad de viviendas además se tomó como caso de comparación un valor promedio de la RMBA a partir de datos publicados en Angel (2016).

Para profundizar en la comparación de los estándares resulta de interés el trabajo realizado en el proyecto Density Atlas del Massachusetts Institute of Technology, en donde con un criterio de similar tipificación de las unidades de análisis, se presentan de manera comparable diferentes datos cuantitativos referentes a la densidad de diferentes casos de estudios teóricos y reales. Para este estudio se tomó el caso teórico de Garden City desarrollado originalmente por Howard Ebenezer, como caso comparativo para la forma de crecimiento definida por Sola Morales de Ciudad Jardín, y el caso de la Villa Radieuse o Radiant City desarrollada por Le Corbusier como caso comparativo de la forma de crecimiento del Polígono.

\subsubsection{Densidad de viviendas}

Es particularmente llamativa la relación entre el valor deseado por la normativa del COT para el Ensanche y los valores que actualmente refleja esta UTC, por el desfasaje entre estado actual y potencial (Gráfico 1). Además, los valores recientemente aprobados por la normativa en el COT se encuentran muy por encima de los valores definidos como deseables por la AEUB lo que plantea una posible tendencia negativa sobre el modelo de ciudad planteado recientemente a nivel local. Las diferencias entre estado actual de las UTC, los estándares de carácter cientificista y los definidos por normativa ponen en evidencia criterios muy disímiles de modelos de ciudad.

El caso del Polígono, respondiendo a una normativa específica, muestra una densidad muy por encima de la normativa de la zona circundante, ya que la densidad se encuentra establecida según el marco teórico de esta forma de crecimiento desde la instancia de proyecto arquitectónico.

Los casos teóricos tomados para el estudio muestran ser más densos por una amplia diferencia tanto en la comparativa Polígono - Radiant City y Ciudad Jardín - Garden City. Estas relaciones permitirían plantear hipótesis para líneas de investigación que relacionen las concepciones teóricas de las formas de crecimiento a través del tiempo, en lo que en principio se apreciaría una tendencia hacia patrones más difusos. 


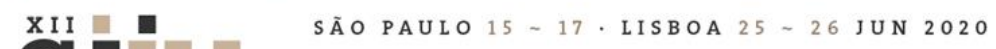 \\ Seminário Internacional de Investigação em Urbanismo \\ Seminario Internacional de Investigación en Urbanismo}

\section{DENSIDAD DE VIVIENDAS}

Estándar 85 Vivienda / Ha

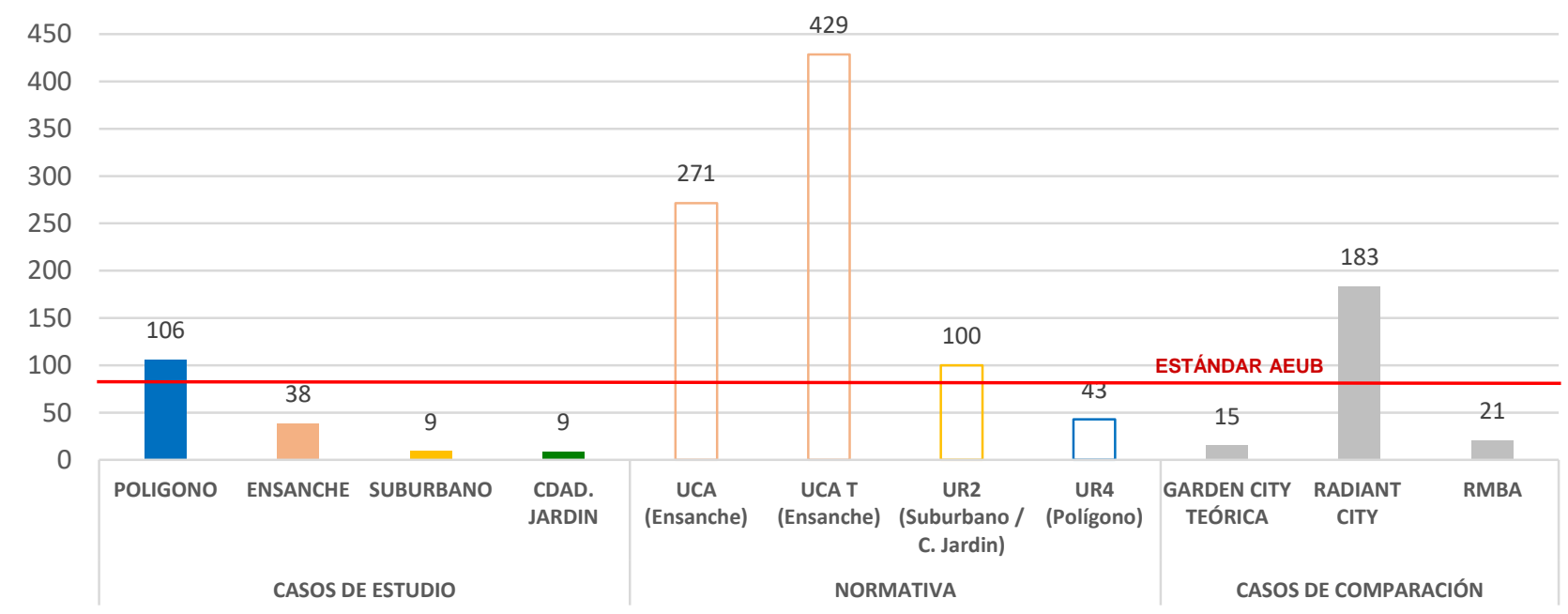

Gráfico 1. Densidad de viviendas. Casos de estudio y de comparación. Fuente: Elaboración propia.

\subsubsection{Compacidad absoluta y compacidad corregida}

La compacidad absoluta muestra valores bajos tanto en los casos de estudio y de comparación (Gráfico 2). De consolidarse la normativa final de los casos de estudio no se alcanzaría los patrones deseables en cuanto uso racional de suelo. El valor definido como estándar es particularmente denso incluso para el caso teórico de la Radiant City. Es llamativo el valor teórico de la Garden City que a pesar de lo que se podría pensarse muestra una densidad relativamente alta en comparación con el resto de los casos.

Los valores de casos de estudio y comparación de la compacidad corregida no alcanzan el estándar deseable (Gráfico 3) incluso con la adaptación propuesta que considera el espacio público total y no el espacio público de estancia. Esto demostraría que, en los casos de estudio, incluso contando con una peatonalización total del viario público, es decir convirtiéndolo a espacio de estancia, no se alcanzaría el estándar propuesto por la AEUB. A pesar de que los casos presentan patrones de edificación poco densos (ver compacidad absoluta), la carencia de espacios públicos no podría habilitar mayor densidad, ya que implicaría una merma en el indicador de compacidad corregida.

La relación entre la densidad ideal por un uso eficiente del suelo y la densidad ideal en relación a la cantidad de espacio público, pone en tensión lo deseable en cuanto uso eficiente de recursos y lo deseable por la población. Este tipo de relación habilitarían una mayor indagación de un indicador que pueda contemplar ambos factores en búsqueda de un estándar ideal ya que la utilización de los indicadores por separado no daría una real idea del desempeño de cada unidad de crecimiento. 


\section{SÃOPAULO15 17 LISBOA 25 26 JUN 2020}

Seminário Internacional de

Seminario Internacional de Investigação em Urbanismo Investigación en Urbanismo

COMPACIDAD ABSOLUTA - Relación entre volumen construido y espacio urbano

Estándar $5 \mathrm{~m}$

6

4

3

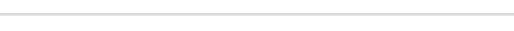

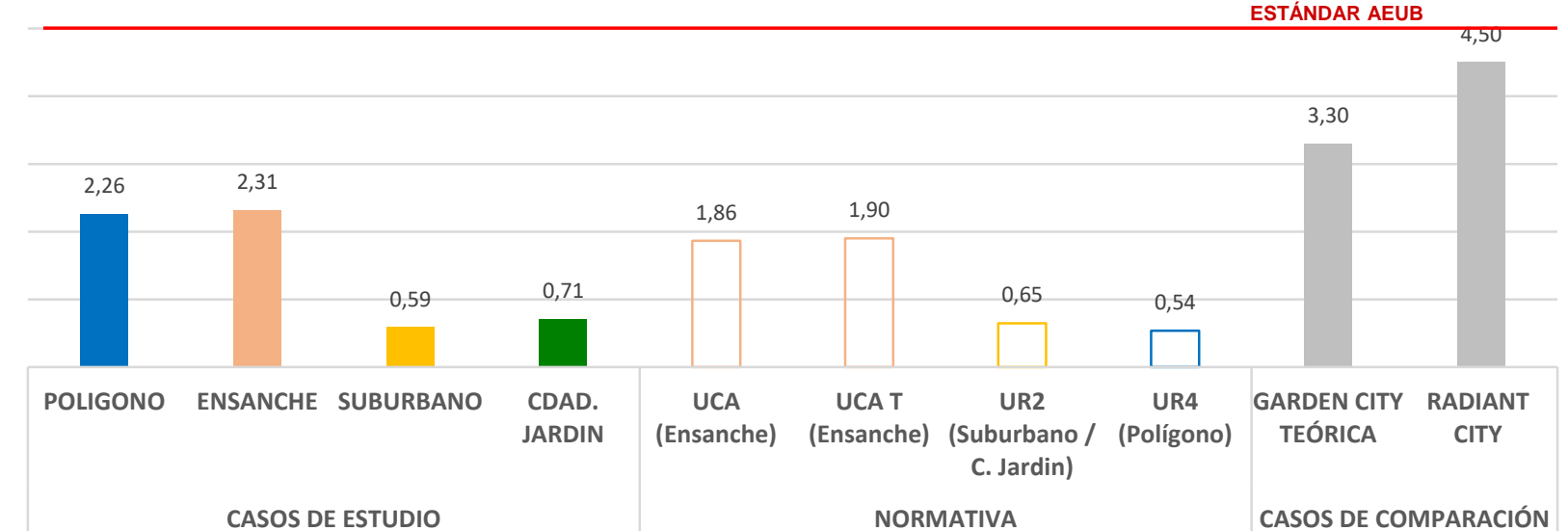

Gráfico 2. Indicador de compacidad absoluta. Casos de estudio y de comparación. Fuente: Elaboración propia.

COMPACIDAD CORREGIDA - Relación entre volumen construido y espacio urbano

Estándar 10 a $50 \mathrm{~m}$

60

50

40

30

20

10

\begin{tabular}{|c|c|c|c|c|c|c|c|}
\hline 6,90 & 9,17 & & & 7,34 & 7,96 & & \\
\hline & & 2,08 & 2,07 & & & 2,30 & 1,64 \\
\hline POLIGONO & ENSANCHE & SUBURBANO & CDAD. JARDIN & UCA (Ensanche) & $\begin{array}{c}\text { UCA T } \\
\text { (Ensanche) }\end{array}$ & $\begin{array}{l}\text { UR2 (Suburbano } \\
\text { / C. Jardin) }\end{array}$ & UR4 (Polígono) \\
\hline \multicolumn{4}{|c|}{ CASOS DE ESTUDIO } & \multicolumn{4}{|c|}{ NORMATIVA } \\
\hline
\end{tabular}

Gráfico 3. Indicador de compacidad corregida. Casos de estudio y de comparación. Fuente: Elaboración propia. 


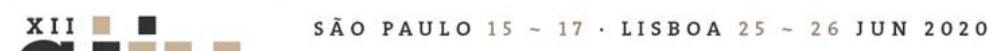

\subsubsection{Permeabilidad urbana}

Como variable ambiental, la permeabilidad sólo es alcanzada por las tipologías más difusas, el suburbano y la ciudad jardín (Gráfico 4). Particularmente, en ninguna de las normativas se alcanza el estándar deseable por la AEUB, por lo que, de aumentar la densidad edilicia de los sectores de estudio, se puede esperar que disminuya el desempeño ambiental por debajo del estándar. En este sentido, la búsqueda de un estándar de densificación debiera establecer criterios entrecruzados de densificación y ocupación del suelo.

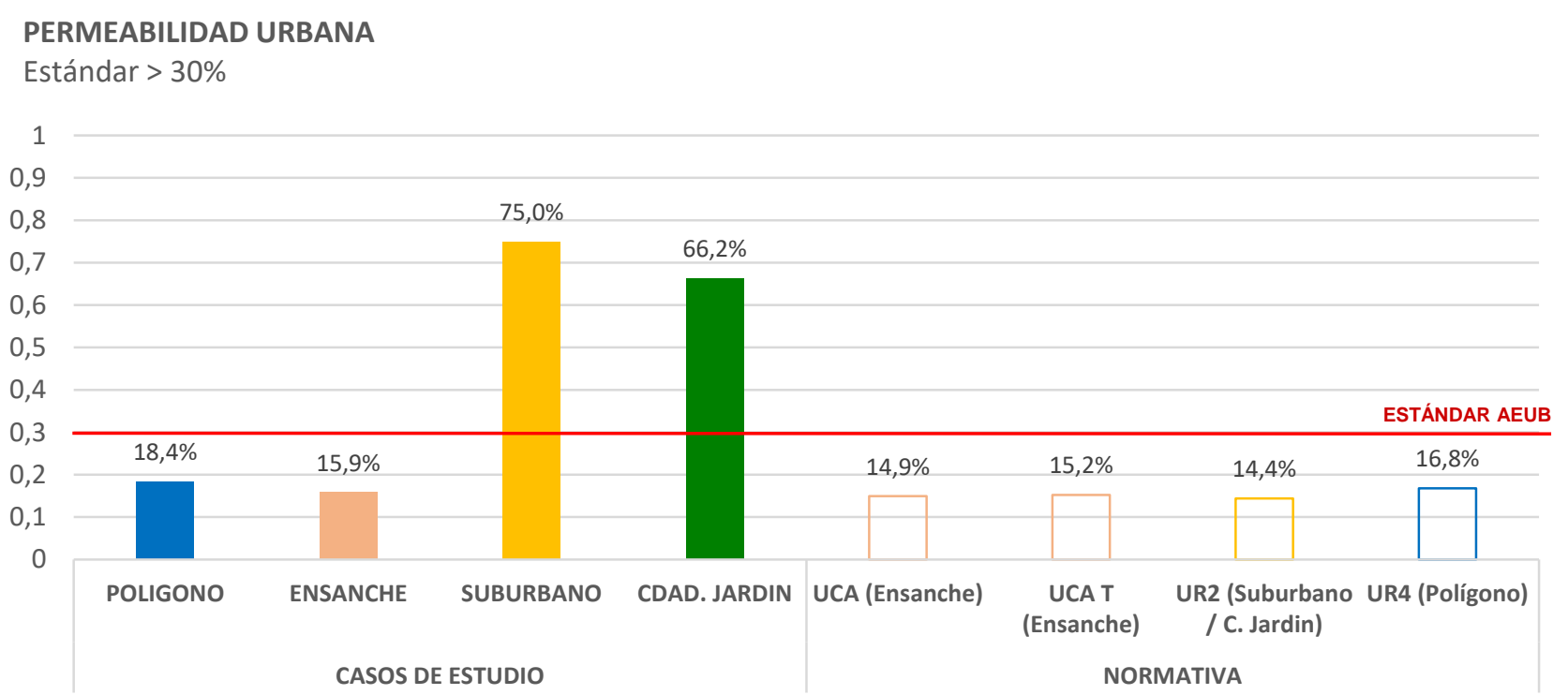

Gráfico 4. Indicador de compacidad corregida. Casos de estudio y de comparación. Fuente: Elaboración propia.

\subsection{Formas de crecimiento y la complejidad de corrección de indicadores}

La evaluación mediante indicadores es una herramienta de análisis cuantitativo que permite un diagnóstico de un sector determinado, en este caso las UTC. Esta herramienta de diagnóstico puede ser aplicada a lo largo del tiempo de manera tal de servir como observatorio de evaluación del crecimiento urbano de cada unidad territorial de crecimiento. Para poder alcanzar los estándares deseados es de interés indagar en las medidas correctivas necesarias para direccionar la tendencia observada en el tiempo hacia un objetivo deseado.

La corrección de estos indicadores no mostraría en principio la misma complejidad en cada sector de estudio. Tomando la clasificación tipológica de las distintas UTC, podemos inferir que en las Ciudades Jardín y los Polígonos (comúnmente barrios cerrados y conjuntos habitacionales) por sus características intrínsecas poseen una mayor complejidad de lo que podríamos denominar tejido tradicional, el Ensanche y el suburbano. Esta hipótesis adquiere mayor importancia en casos como el municipio del Pilar donde las Ciudades Jardín ocupan gran proporción del territorio (Figura 10). De esta forma se podría identificar de manera expeditiva las áreas de mayor complejidad para corrección de indicadores. 


\section{SÃOPAULO15 $17 \cdot$ LISBOA $25 \sim 26$ JUN 2020}

\section{Conclusiones y líneas abiertas de investigación}

La amplia diferencia entre estándares establecidos con criterios cientificistas, en este caso por la AEUB, y los estándares establecidos por gobiernos locales mediante normativa, pone en evidencia la disociación de objetivos en cada caso. En este sentido, ni los resultados presentes en la actualidad, ni la normativa, ni los estándares europeos parecen ser pertinentes por lo menos en los casos de estudio trabajados. Se plantea la necesidad de indagación de estándares que entrecrucen densidades edilicias, espacio urbano, espacio público y variables ambientales.

Los criterios de análisis de sustentabilidad mediante indicadores podrían no ser iguales dependiendo del estado de densificación, por lo que las estrategias de corrección también debieran diferir. La clasificación de las UTC según densidad y consolidación adquiere relevancia ya que se podría hacer una categorización para establecer estas acciones correctivas en función de la complejidad de cada sector.

Las relaciones entre indicadores, normativa y nivel de consolidación abren nuevas preguntas de investigación. ¿Cuál es la saturación de normativa pertinente que habilitaría la expansión territorial? ¿Cómo operar una UTC que se encuentran completa en su normativa?; en un contexto de debate de densidad dispersión no resuelto y de estándares de comparación muy disímiles de ¿cuál sería una aproximación al estándar adecuado de densidad urbana?

\section{Bibliografía}

ANGEL, S. et al. (2016). Atlas of Urban Expansion. Edition, Volume 1: Areas and Densities. New York: New York University, Nairobi: UN-Habitat, and Cambridge, MA: Lincoln Institute of Land Policy.

CÓDIGO DE ORDENAMIENTO TERRITORIAL (2019) Municipalidad del Pilar. Partido del Pilar

COMPAGNONI, A. M. et al. (2018). Adaptación de un modelo valorativo de sustentabilidad urbana como aporte al conocimiento del hábitat urbano. XXXII Jornadas de Investigación SI FADU UBA. Buenos Aires, 27 y 28 de septiembre.

DEMOGRAPHIA (2019). World Urban Areas (Built Up Urban Areas or World Agglomerations) 15th annual edition.

DIEZ, F. (1996). Buenos Aires y algunas constantes en las transformaciones urbanas. Buenos Aires: Editorial de Belgrano.

FARGUELL, X. M. (2007). Reflexiones sobre la ciudad: más allá de la ciudad difusa, más allá de la ciudad densa. En F. INDOVINA (comp.) La ciudad de baja densidad. Lógicas, gestión y contención. Barcelona: Diputación de Barcelona.

GARCÍA, A. (1999). Modelo de ciudad, modelo de producción. En A. GARCIA y S. RUEDA (comps.) La ciudad sostenible. Barcelona: Centro de Cultura Contemporánea de Barcelona.

LANFRANCHI, G. et al. (2018). ¿Cómo crecen las ciudades argentinas? Estudio de la expansión urbana de los 33 grandes aglomerados. Buenos Aires: CIPPEC. 


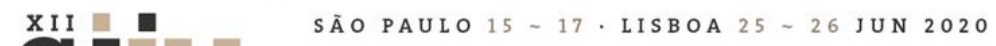

POSKUS, M. et al. (2018). Desafíos de la vivienda: El Acceso a la ciudad. Nuevas Metodologías para caracterizar el déficit urbano-habitacional. En V. ADLER y F. VERA (comps.), Vivienda ¿Qué viene? De pensar la unidad a construir la ciudad (102-240). BID.

RUEDA, S. (2010). El urbanismo ecológico. Barcelona: Agencia de Ecología Urbana de Barcelona. — (2018). Certificación del urbanismo ecosistémico. Barcelona: Agencia de Ecología Urbana de Barcelona. - (Enero de 2018). Las supermanzanas, base del modelo funcional, urbanístico y social de una ciudad. Gobierno de la Ciudad de Buenos Aires. Ciudad de Buenos Aires.

SOLA MORALES, M. (1997). Las formas de crecimiento urbano. Barcelona: Universidad politécnica de Catalunya.

UN-HABITAT (2017). Nueva Agenda Urbana.

Fuente electrónicas

http://densityatlas.org (consulta: 01/02/2020) 